\title{
Microstructure Evolution of Sol-gel Derived Oxide Nanostructures with In- Situ TEM
}

\author{
Aiming Yan* and Vinayak P. Dravid* \\ *Department of Materials Science and Engineering, Northwestern University, Evanston, IL \\ 60208, USA
}

Solution-derived (oxide) ceramics are widely used in sintering of thin/thick films, multilayer, fine-grained nanocrystalline powders or ceramic composites. The sintering trajectories for constrained films or nanocrystalline ceramic materials are known to differ from the bulk or coarse-grained ceramic materials due to the substrate constraints and reduced grain size. A number of experimental and theory computational studies have been carried out to study the sintering of thin films or nanocrystalline ceramic materials under the constraints from substrate or external stress ${ }^{1-4}$, but seldom are there reports on conversion of nanoscale precursor to solidstate contiguous form. In contrast to thin/thick films or multilayers, the conversion of nanoscale precursors (e.g., 0-D dots, 1-D lines) involves additional thermodynamic and kinetic factors due to high surface-to-volume ratio of the nanostructures, interfacial and surface areal influence which introduces extra stresses, and symmetry and lattice mismatch considerations with respect to the substrates - all of which further complicates the internal microstructure and external shape evolution during the conversion processes 5,6 .

In the present study, cobalt ferrite $\left(\mathrm{CoFe}_{2} \mathrm{O}_{4}\right.$ or $\left.\mathrm{CFO}\right)$ nanostructures were fabricated by the SofteBL approach ${ }^{6}$, involving spin-coating CFO sol-gel onto eBL pre-patterned substrates. This results in site-/shape-specific and three-dimensionally constrained oxide nanostructures on variety of substrates, ranging from amorphous $\mathrm{SiNx}$ membrane (amenable to in-situ $\mathrm{S} / \mathrm{TEM}$ ) to single-crystal substrates for single crystal conversion. CFO thin films and nanopatterns were investigated by in-situ S/TEM thermal annealing to probe the nature of nucleation and grain growth of nanostructures under dimensional and spatial constraints. We find differential growth mechanisms which dominate at different annealing regime.

The initial results show that CFO thin films and nanopatterns on amorphous SiNx membrane undergo abnormal grain growth (Figure 1) under specific annealing conditions. On the other hand, CFO thin films grown on single-crystal $\mathrm{MgO}$ substrate result in a preferred orientation defined by the crystal orientation of the substrate (Figure 2), which allows examination of epitaxial growth mechanism of thin films on single-crystal substrates. Utilizing in-situ TEM characterization, the grain growth mechanisms in different nanostructure systems can be understood, and the role of different constraint factors such as spatial constraint due to the substrate, dimensional constraint due to nanoscale size of the patterns, and lattice mismatch between the nanostructure and the substrate, are revealed. The presentation will cover strategies for in-situ S/TEM of thin-film patterned nanostructures on electron transparent substrates as model geometries without the need for invasive specimen preparation processes. 


\section{References:}

1. $\quad$ Bordia, R. K.; Scherer, G. W. Acta Metallurgica 1988, 36, (9), 2411-2416.

2. Kumar, K. P.; Keizer, K.; Burggraaf, A. J.; Okubo, T.; Nagamoto, H. Journal of Materials Chemistry 1993, 3, (11), 1151-1159.

3. $\quad$ Martin, C. L.; Bordia, R. K. Acta Materialia 2009, 57, (2), 549-558.

4. $\quad$ Stech, M.; Reynders, P.; Rodel, J. Journal of the American Ceramic Society 2000, 83, (8), 18891896.

5. $\quad$ Pan, Z.; Li, S.; Wang, Z.; Yu, M. F.; Dravid, V. P. Applied Physics Letters 2007, 91, (14).

6. $\quad$ Pan, Z. X.; Alem, N.; Sun, T.; Dravid, V. P. Nano Letters 2006, 6, (10), 2344-2348.

7. This work was supported by the U.S. Department of Energy (DOE-BES) programs, the grant number DE-FG02-07ER46444. DOE's support does not constitute an endorsement by DOE of the views expressed in the article. The research work was performed in the EPIC facilities of the NUANCE Center at Northwestern University and NCEM in Lawrence Berkeley National Lab. NUANCE Center is supported by NSF-NSEC, NSFMRSEC, the State of Illinois, and Northwestern University. NCEM is supported by the Office of Science, Office of Basic Energy Sciences of the U.S. Department of Energy under Contract No.DE-AC02-05CH1123.

(a)

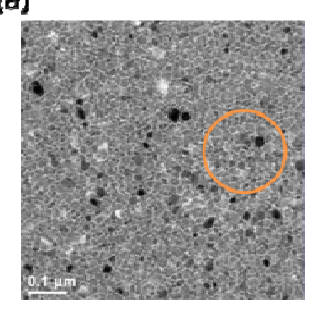

13(min):48(sec).53(1/100sec)

(d)

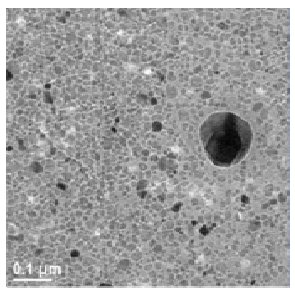

$15: 21.82$ (b)

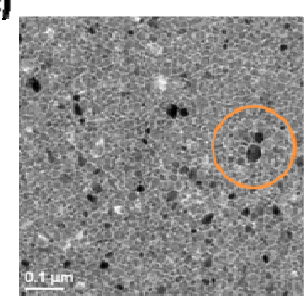

$13 ; 48.83$

(e)

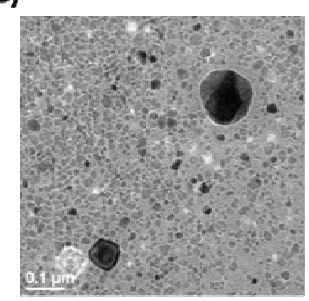

15:38.39 (c)

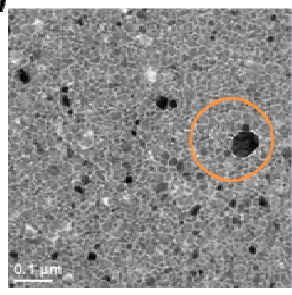

13:52.16

(f)

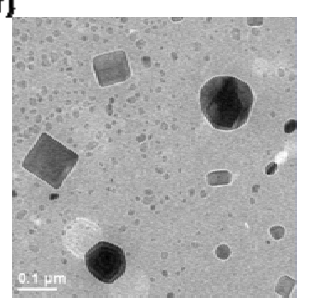

17: 07.14
Figure 1: (a) before grain coalescence (circled area is where the first island started to grow; (b) the first island nucleated and started to grow; (c) (d) the first island kept growing; (e) the second island started to grow; (f) more islands during the coalescence. Scale bar: 0.1 $\mu \mathrm{m}$.
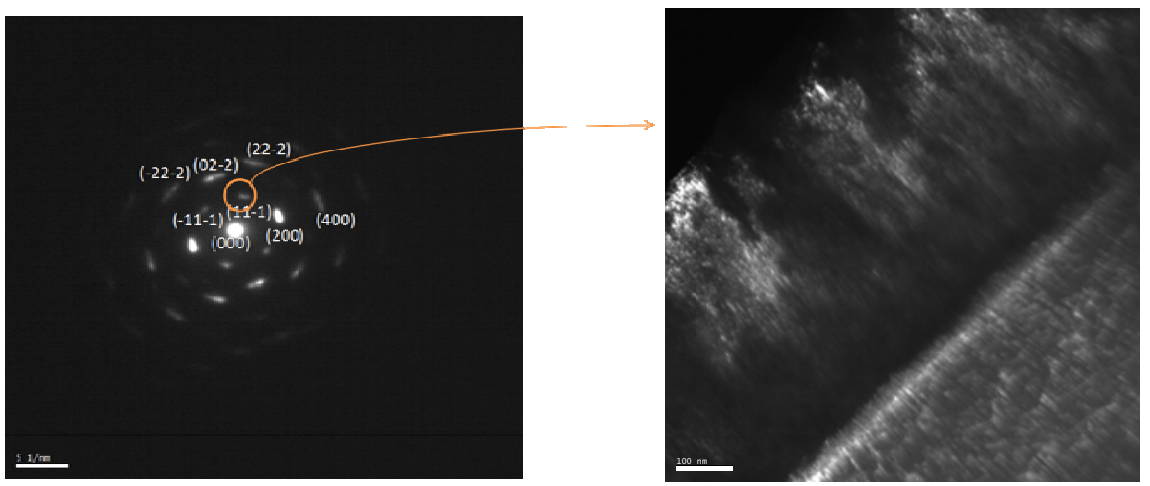

Figure 2: (left) diffraction pattern of CFO film on MgO single-crystal substrate (011); (right) dark field image from one dim diffraction spot in the diffraction pattern on the left (scale bar: 100nm). 